\title{
AADL-Based Flight Management System Modeling
}

\author{
Jiawei Zheng ${ }^{\text {a) }}$, Lichen Zhang \\ School of Computer, Guangdong University of Technology, Guangdong 510006, China. \\ a) Corresponding author: 1925584561@qq.com
}

\begin{abstract}
Flight management system is an important part of avionics system. Traditionally, the schedulability analysis of this system was carried out after the design of the system was completed and in the stage of implementation and verification, this made the system unable to accurately analyze the hardware and software requirements. Modeling with advanced modeling method AADL provides possibilities for schedulability analysis, reliability analysis, and communication delay analysis of the flight management system, making it possible to accurately determine the hardware and software requirements of the system during the system requirements analysis phase, and can greatly reduce the system's change verification costs.
\end{abstract}

Key words: Flight Management System; AADL Modeling.

\section{INTRODUCTION}

With the rapid economic growth, civil aviation has developed rapidly. The fleet size, the number of airports and flights have continued to increase. The application of flight management systems allows airlines to get a reasonable schedule, and ultimately gives the most concise and most efficient plan scheme to improve the current state of air traffic management,

The Flight Management System(FMS) is a computer system that integrates multiple on-board electronic systems. It provides flight time, distance, speed, and altitude predictions that reduce cockpit workload, improve efficiency, and eliminate Many daily operations that were conventionally performed by the driver made the aircraft safe and economical to fly [1].

Based on the ARINC 702A standard, the main functions provided by the flight management system are: navigation, flight planning, track prediction, performance calculation, guidance.

Architecture Analysis and Modeling Language (AADL) is a text and graphics language [2], which designed and analyzed for performance-critical real-time embedded system hardware and software architectures, suitable for time, throughput, reliability, security, and The use of embedded systems that are subject to restrictions such as confidentiality, are often used in areas such as aerospace, aerospace, automotive and autonomous systems. AADL can provide hardware platforms such as processor, memory, bus, and device for modeling, processes, and threads.

\section{The Role of FMS at Various Stages of Flight}

1)Take-off phase: When the pilot is preparing for take-off, the flight management computer (FMC) calculates the flight path through the flight plan entered by the FMC's control display unit (CDU). The FMC calculates the flight path according to the aircraft's full weight, cruising altitude, reserve oil level, and outside temperature input on the CDU. For performance data, FMC calculates the best takeoff target thrust of the aircraft and the vertical profile of the aircraft flight.

2) Climb phase: The climb phase generally refers to the take-off altitude to the cruising altitude. The take-off altitude of the aircraft is $400 \mathrm{ft}$. After the pilot turns on the lateral navigation (L-NAV) and vertical navigation (VNAV), the FMS makes horizontal and vertical navigation of the aircraft according to the flight path, and determines 
the target thrust and target speed according to the pilot's choice, provides the best climbing profile (the best climb angle and climb speed), and recommends the height of the segmented climb and climb vertices.

3) Cruise phase: FMS chooses the best cruising speed and best cruising altitude according to the length of the route and the route conditions. Use the path of the large-circle route between the airport of departure and the airport of destination.

4) Fall phase: The fall phase generally refers to the cruising altitude to the entry altitude. The FMS determines the starting point of the descent of the aircraft based on the data stored in the pilot input and navigation database, determines the descent speed during the descent, and makes maximum use of the aircraft's altitude potential energy. To save fuel.

5) Approach phase: The approach phase generally refers to the aircraft's approach altitude and safe landing altitude. When the aircraft is at the end of the descent, FMS will calculate the optimal speed to guide the aircraft to the runway entrance and landing point.

\section{Functional Implementation of FMS}

1) Navigation function: The navigation function is used to determine the position of the aircraft at the time [3], to perform navigation calculations, and to automatically adjust the navigation station management. Completion of the aircraft transverse profile flight management, guide the aircraft to arrive at the destination according to the scheduled route, including: automatic selection of navigation, automatic adjustment and IRS calibration, starting from the airport to take off, according to the destination airport to be selected to determine the location of the route, Distance, time, speed, etc. from the destination or flight route point.

2) Performance management function: During the flight, calculate the relevant performance indicators of the aircraft, namely the flight altitude, speed, climb, descent, climb speed, and descent speed of the aircraft to obtain the best vertical preselected flight path and complete the longitudinal section management of the aircraft. FMC's performance calculations are based on benchmark data provided by the performance database, some signal data from external sensors, required driver input on the CUD, and parameter limit values.

3) Guidance function: Guidance is a decision made when an aircraft flies along pre-selected tracks and is subject to disturbances or navigation uncertainty causing deviations from pre-selected tracks. The It divided into horizontal guidance and vertical guidance. Horizontal guidance is to control the deviation of the actual track from the pre-selected path in the horizontal plane according to a certain control rate. When the horizontal guidance function is turned on, the FMC provides a heading control command to control the aircraft to fly along pre-selected routes. Vertical guidance is based on a certain vacancy rate to control the actual track in the vertical plane relative to the pre-selected track deviation. When the vertical guidance function is on, the FMC provides speed and lift command to control the flight of the aircraft along a preselected longitudinal path.

4) Flight plan management: The flight plan is input to the FMS through the MCDU, it includes the horizontal trajectory to be flown (horizontal flight plan) and the vertical trajectory to be flown, a speed and altitude profile (vertical flight plan). This information is necessary for proper performance calculations and guidance instructions. The guidance section of the FMC stores horizontal and vertical sections of cross-section data. After calculating the position of the aircraft and comparing it with the actual position of the aircraft, it generates instructions for inputting aircraft control calculations and thrust management computers based on the error between them. Then, the flight control computer and the thrust management computer generate the actual steering command and the automatic throttle thrust command to operate the aircraft to realize the automatic driving control of the flight route by the FMC.

5) Database management functions: The flight management system has a performance database and a navigation database. The performance database is the basis for aircraft performance management and is used to perform performance optimization calculations to ensure that the aircraft flies along the optimal vertical profile. The navigation database is designed to provide automatic navigation capabilities for the aircraft from take-off to landing. It stores navigation information for the entire area, including the airport, the waypoints, the navigation station's geographical position, frequency, and the route composition structure of the aircraft flight area.

6) Advisory and alarm functions: Pilots can obtain many consulting information through the CDU, such as flight profile related information, performance related information and system failure information, etc. At the same time, FMS can also provide automatic alarm functions, such as wind shear warning, near-ground warning, TCAS warning and so on. 


\section{Flight Management System Modeling Analysis}

AADL can be used to model and analyze systems that are in use [4], can design and integrate systems, can be used for analysis of partially defined modules, and can also be used for analysis of complete systems extracted from source code. AADL supports early prediction and analysis of performance-critical system quality.

Based on the functional analysis of the flight management system, the flight management system can be divided into six processing threads, which are navigation sensor processing threads, integrated navigation threads, guidance threads, flight planning threads, aircraft performance optimization threads, and control display threads. The flight management system mainly uses periodic tasks to complete the modeling [5]. refers to that punishes the task according to a certain time interval. The mathematical model of the periodic task set $\tau$ is represented as $\tau=\{\varphi, E, D, T\}$, where $\varphi$ is the initial request time, $\mathrm{E}$ is the task execution time, $\mathrm{D}$ is the relative dead line, and $\mathrm{T}$ is the task cycle. The tasks of the flight management system discussed in this paper are $\varphi=0$ and $\mathrm{D}=0$. Therefore, the task model is simplified as $\tau=\{C, D\}$. The six mission models of the flight management system are TNSP $=(15,50)$, TIN $=(40,100), T G P=$ $(30,50)$, TFP $=(30,200)$, TAP $=(100,500)$, THP $=(1,50)$, in $\mathrm{ms}$.

The communication between tasks uses a shared data area. In order to avoid the producer-consumer problem of the shared data area, the communication between the tasks is simplex. The AADL modeling for the six threads of the flight management system is as figure1.

\section{Feasibility Analysis of the Model}

Security is of vital importance to the aviation industry. Therefore, feasibility analysis of real-time systems during the system design phase is necessary. The flight management system modeling used in this article is a periodic task. This section mainly discusses the schedulability analysis of the periodic task set $\tau$. For the periodic task set $\tau$, if each dead line of each task can be satisfied under one schedule $S$, the schedule $\mathrm{S}$ is said to be a valid schedule for the task set. For a $\tau$, if there is a valid schedule, the periodic task is said to be feasible. If there is an algorithm that can generate a valid schedule, the periodic task set $\tau$ is said to be feasible under the schedule algorithm.

For dynamic priority scheduling algorithms, the best-known is Earliest Deadline First (EDF),

which is used in real-time operating systems to place processes into a priority queue. Whenever scheduling time occurs, the queue is searched for the process that is closest to the deadline. Through the scheduling period is equal to the period of its regular process, EDF has a $100 \%$ utilization limit, as long as the total CPU utilization does not exceed $100 \%$, you can ensure that all deadlines can be met. But when the system is overloaded, the set of processes that will miss the last deadline is largely unpredictable, which is a considerable disadvantage for real-time system design.

For fixed-priority scheduling algorithms, the most famous are Rate-Monotonic (RM) and Deadline-Monotonic (DM) algorithms. The RM algorithm assigns the highest priority to the task with the smallest period, and the DM algorithm gives the task with the lowest relative deadline the highest priority. When the period of all tasks in the periodic task set system is equal to the relative dead line, DM and RM are equivalent. For single-processing systems, the DM algorithm is the optimal algorithm. When the dead line of some tasks is not equal to the period, the RM algorithm is the optimal algorithm. If the real-time task system consists of $n$ tasks, the relative dead line of each task is equal to the period, which is settable by the DM or RM.

When the task of the flight management system is 6 , the utilizable rate of the system is $73.4 \%$.

Figure 2 shows the relationship between processor performance and system utilization for the AADL modeling of a flight management system under speed monotonic scheduling algorithms. It can be seen from the graph that there is a linear relationship between processor performance and load. According to the simulation results, it can be concluded that the flight management system must be able to meet the six tasks schedulability requirements under the processor cycle time $<450 \mathrm{ps}$, that is, the utilization rate is less than $73.4 \%$. In some real-time security-critical system implementations, certain system margins need to be ensured in order to ensure system reliability and fault tolerance. The above simulation analysis based on the AADL model of the flying tube system provides the basis for processor selection, system design, software design and optimization, and it can achieve the optimal balance between system, hardware and software implementation costs [6]. 
Thread NavigationSensorProcessing Properties

Dispatch_Protocol $=>$ Periodic;

Period = > $50 \mathrm{~ms}$;

Compute_Excution_Time $=>15 \mathrm{~ms}$

End NavigationSensorProcessing

Thread GuidanceProcessing

Properties

Dispatch_Procotol => Periodic;

Period => $50 \mathrm{~ms}$;

ComputeExcution_Time $=>30 \mathrm{~ms}$;

End GuidanceProcessing;
Thread IntegratedNavigation

Properties

Dispatch_Protocol $=>$ Periodic;

Period $=>100 \mathrm{~ms}$;

Compute_Excution_Time $=>40 \mathrm{~ms}$;

End IntergratedNavogation

Thread FlightPlanProcessing

Properties

Dispatch_Procotol $=>$ Periodic;

Period => $200 \mathrm{~ms}$

Compute_Excution_Time $=>30 \mathrm{~ms}$;

End FlightPlanProcessing;

Thread AircraftPerfomanceCalculation

Properties

Thread HandlePageRequest

Properties

Dispatch_Procotol => Periodic;

Dispatch_Procotol => Periodic;

Period $=>500 \mathrm{~ms}$;

Compute_Excution_Time $=>1 \mathrm{~ms}$;

Compute_Excution_Time $=>100 \mathrm{~ms}$;

End AircraftPerformanceCalculation;

End HandlePageRequest;

Processor implementation PowerPC.Mhz500

Properties

SEI ::cycle_time => $700 \mathrm{ps}$;

End PowerPC.Mhz500

FIGURE 1. AADL Modeling of Flight Management System

$$
\begin{aligned}
& U_{s u n}(T)=\sum_{i=1}^{n} \frac{e i}{p i} \leq\left(2^{\frac{1}{n}}-1\right) \\
& \lim _{n \rightarrow \infty} \sum_{i=1}^{n} \frac{e i}{p i} \leq n\left(2^{\frac{1}{n}}-1\right) \approx 0.69
\end{aligned}
$$




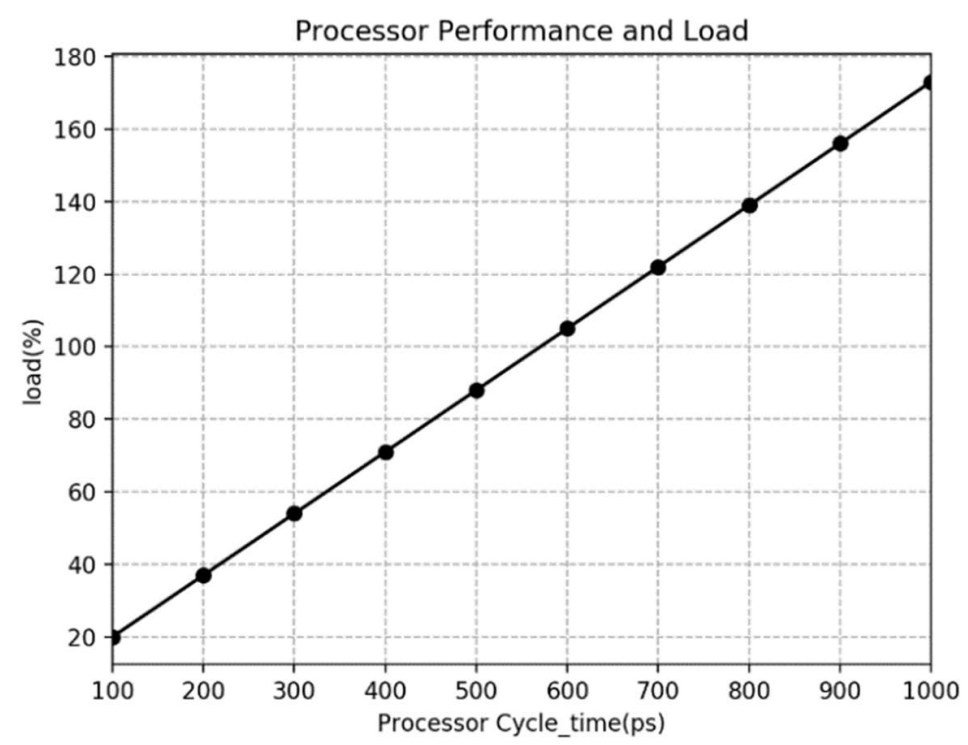

FIGURE 2. Processor Performance and Load

\section{CONCLUSION}

With the rapid development of civil aviation, the scale of the aircraft has been expanded, and the application of the flight management system will allow the airline's aircraft to be properly dispatched. The role of the flight management system in each flight phase and its function implementation are introduced. Based on the AADL, the flight management system is modeled, and the schedulability is further evaluated and analyzed. In order to solve some complex task assignments, further research is needed.

\section{ACKNOWLEDGMENTS}

This work is supported by the national natural science foundation of China under grant (No.61572142), natural science foundation of Guangdong province under grant (No.2015A030313490).

\section{REFERENCES}

1. YIN Fengshe, TANG Xiaoming. research of avionics system modeling based on AADL [J]. Ship Electronic Engineering, 2013(4):88-89.

2. Han-bo Wang, Xing-she Zhou, Yun-wei Dong, Research on architecture analysis and design language [J]. Computer Engineering and Applications, 2009(16):1-4.

3. Zhong Wei, Lichen Zhang. Flight Guidance and Control System Modeling and Design Based on AADL [C]. 2016 2nd Workshop on Advanced Research and Technology in Industry Applications, 2016: 436-440.

4. Weitao Miao. Research on Design Method of General Aircraft Flight Management System [c]. Civil Aircraft Aviation International Forum,2016(6):228-233.

5. SAE International. Flight Management Systems Review [ M], 2007.

6. Singhoff F, Legrand J,Nana, L,et.Scheduling and Memory Requirements Analysis with AADL. ACM SIG Ada Letters, 2005. 